\title{
Exposure-Response and Tumor Growth Inhibition Analyses of the Monovalent Anti-c-MET Antibody Onartuzumab (MetMAb) in the Second- and Third-Line Non-Small Cell Lung Cancer
}

\author{
Kelong Han, ${ }^{1,6}$ Pascal Chanu, ${ }^{2}$ Fredrik Jonsson, ${ }^{3}$ Helen Winter, ${ }^{4}$ René Bruno,, 5 Jin Jin,,${ }^{4}$ and Mark Stroh ${ }^{4}$
}

Received 3 July 2016; accepted 12 December 2016; published online 27 December 2016

\begin{abstract}
The phase III trial comparing onartuzumab + erlotinib vs. erlotinib in the second- and third-line non-small cell lung cancer (NSCLC) did not meet its primary endpoint of overall survival (OS). The objective was to assess whether doses higher than the phase III dose $(15 \mathrm{mg} / \mathrm{kg})$ might yield better efficacy without compromising the safety profile. Data were from 636 patients from the phase II and III NSCLC studies. Tumor growth inhibition (TGI) models were fit to longitudinal tumor size data to estimate individual TGI metrics including time to tumor re-growth (TTG). Cox regression models were developed for timeto-event endpoints (progression-free survival (PFS), OS, and TTG) to investigate relationships with baseline prognostic factors and onartuzumab exposure. Incidence of adverse events was modeled by logistic regression. In the final models, higher onartuzumab exposure was associated with longer PFS, but not with longer OS. Longer OS was associated with higher baseline albumin, longer TTG, smaller number of metastatic sites, female gender, lower ECOG score, and younger age. TTG was the only TGI metric retained in the final OS model. Onartuzumab exposure was not significantly associated with TTG after adjusting for prognostic factors. Higher Cmin was associated with increased incidence of infusion reactions and peripheral edema. Higher onartuzumab exposure was not significantly associated with improved OS after adjusting for prognostic factors and TTG, and there was a trend of unknown clinical significance toward increased incidence of infusion reactions and peripheral edema. These results did not support testing higher onartuzumab doses.
\end{abstract}

KEY WORDS: Cox regression; exposure-response; NSCLC; onartuzumab; survival model; time to tumor re-growth; tumor growth inhibition.

\section{INTRODUCTION}

c-MET is a receptor tyrosine kinase and plays a key role in a variety of cellular processes including motility, morphogenesis, proliferation, and survival and invasion and may also contribute to angiogenesis (1). Clinically, the c-MET pathway has been strongly linked to oncogenic potential. First,

Electronic supplementary material The online version of this article (doi:10.1208/s12248-016-0029-5) contains supplementary material, which is available to authorized users.

\footnotetext{
${ }^{1}$ Clinical Pharmacology Modeling \& Simulations, GlaxoSmithKline,

709 Swedeland Rd, King of Prussia, Pennsylvania 19406, USA.

${ }^{2}$ Clinical Pharmacology, Genentech/Roche, Lyon, France.

${ }^{3}$ qPharmetra, Stockholm, Sweden.

${ }^{4}$ Clinical Pharmacology, Genentech Inc., South San Francisco, California, USA.

${ }^{5}$ Clinical Pharmacology, Genentech/Roche, 84 Chemin des Grives, 13013, Marseille, France.

${ }^{6}$ To whom correspondence should be addressed. (e-mail: kelong.x.han@gsk.com, rene.bruno@roche.com)
}

activating kinase domain mutations have been described in a variety of cancers, with particularly high levels observed in renal papillary carcinoma patients $(2,3)$. Second, high levels of tumoral expression of c-MET and/or its ligand hepatocyte growth factor (HGF) have been correlated with worse prognosis in several tumor types, including non-small cell lung cancer (NSCLC), breast cancer, ovarian cancer, cervical cancer, gastric cancer, transitional bladder carcinoma, glioblastoma, head and neck cancers, and multiple myeloma (413). Collectively, these provide compelling evidence that therapeutics abrogating c-MET activation warrant clinical evaluation.

Onartuzumab (MetMAb) is a recombinant humanized monoclonal monovalent anti-c-MET antibody that binds the extracellular domain of c-MET, blocking HGF ligand binding, and inhibiting subsequent receptor activation. In a randomized placebo-controlled phase II study in recurrent NSCLC (OAM4558g, ClinicalTrials.gov Identifier: NCT00854308), onartuzumab plus erlotinib significantly improved progression-free survival (PFS) and overall survival (OS) as compared to erlotinib plus placebo in MET-positive (MET 
immunohistochemistry diagnostic positive) patients (14). However, in a double-blind randomized placebo-controlled phase III study in recurrent NSCLC (OAM4971g, ClinicalTrials.gov Identifier: NCT01456325), onartuzumab plus erlotinib did not significantly improve PFS or OS as compared to erlotinib plus placebo in MET-positive patients (15).

The onartuzumab dose used in the phase III study, $15 \mathrm{mg} / \mathrm{kg}$ administered once every 3 weeks (Q3W), was supported by cumulative preclinical and clinical experience. Simulations based on a population pharmacokinetic (PK) model derived from phase I study OAM4224g and phase II study OAM4558g suggested that onartuzumab $15 \mathrm{mg} / \mathrm{kg}$ Q3W would achieve a target tumoristatic trough concentration of $15 \mu \mathrm{g} / \mathrm{mL}$, which was derived from xenograft studies (16). However, OAM4224g showed that onartuzumab was tolerable up to $30 \mathrm{mg} / \mathrm{kg}$ (17), opening up the possibility of dose intensification.

In order to facilitate the interpretation of the phase III outcomes and assess whether further dose intensification might yield better efficacy without compromising the safety profile, exposure-response analyses were conducted. However, exposure-response models may be confounded by disease severity and prognostic factors that may be observed (e.g., albumin, ECOG score, number of metastatic sites) or unobserved (e.g., inflammatory status). Confounding is related to the fact that disease severity and health status may impact both OS and PK $(18,19)$, especially for monoclonal antibodies. One way to address the confounding issue is to incorporate an explanatory variable, such as TGI metrics as illustrated in the causal pathway in Fig. 1 (18) to make inferences about OS.

The objectives of these analyses were to (1) assess the relationship between onartuzumab exposure and efficacy as well as safety and (2) estimate TGI metrics and assess onartuzumab exposure-TGI and TGI-efficacy relationships (18).

\section{METHODS}

\section{Patients and Exposure Metrics}

Onartuzumab PK, efficacy, and safety data were obtained from OAM4558g (14) and OAM4971g (15). The clinically relevant covariates tested included those related to demographics, laboratory tests, concomitant medications, and pathophysiological factors (Table I). Onartuzumab serum

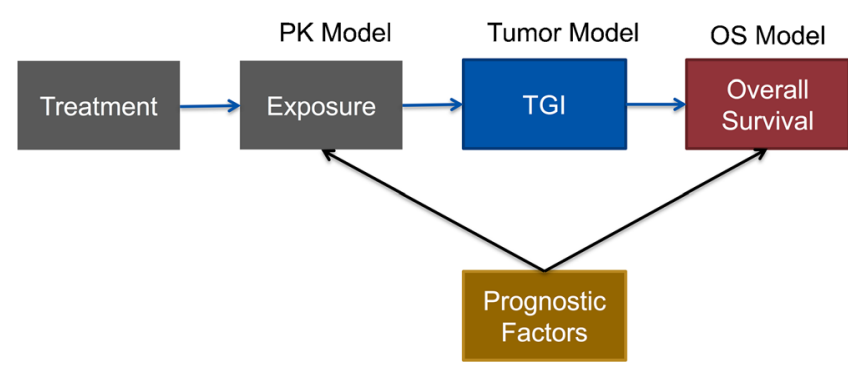

Fig. 1. Causal pathway to the elicitation of treatment effect. TGI tumor growth inhibition. TGI metrics includes the growth rate constant (KG) (24), the time to tumor re-growth (TTG) (25), and tumor size ratio to baseline (25) concentrations were determined at Genentech Inc. by a validated sandwich enzyme-linked immunosorbent assay described previously that was designed to measure free onartuzumab (16). The lower limit of quantification (LLOQ) was $0.2 \mu \mathrm{g} / \mathrm{mL}$.

Erlotinib was administered at the approved daily oral dose of $150 \mathrm{mg}$. Onartuzumab was administered at $15 \mathrm{mg} / \mathrm{kg}$ via intravenous infusion once every 3 weeks. Per study protocol, patients in the erlotinib arm were allowed to cross over to the onartuzumab + erlotinib arm after disease progression. A population PK model of onartuzumab (twocompartment model with linear elimination) has been previously established (16) based on data from phase I and II studies. The estimates for clearance (CL), central volume of distribution (V1), and terminal half-life were $0.439 \mathrm{~L} / \mathrm{day}$, $2.77 \mathrm{~L}$, and 13.4 days, respectively. Onartuzumab CL increased with creatinine CL. Onartuzumab V1 increased with body weight and was higher in males. Onartuzumab peripheral volume of distribution increased with body weight. Exposure of onartuzumab or erlotinib was not associated with MET diagnostic status (degree of tissue MET expression). No pharmacokinetic drug-drug interaction was observed between onartuzumab and erlotinib.

Non-linear mixed effects modeling was performed with NONMEM (version 7.3; ICON Development Solutions, Ellicott City, Maryland, USA) (20) using the FOCE method of estimation with interaction, Perl-speaks-NONMEM (version 3.5.3; Uppsala University, Uppsala, Sweden) (21), and R 3.0.1 (22). Serum concentration data were fit to the previously published population PK model (16) to obtain individual empirical Bayesian estimates of PK parameters (post hoc step using NONMEM (20)), which were subsequently used to derive the individual onartuzumab exposure (trough (Cmin) and peak (Cmax) concentration and area under the concentration time curve (AUC)) during the first dosing interval and at steady state (fifth dosing interval). AUC was calculated using the trapezoidal method. Patients in the erlotinib singleagent arm were assumed to have an onartuzumab exposure of zero. Exposure was tested both as continuous variable and categorical variable (quartiles and tertiles).

\section{Longitudinal Tumor Size Modeling}

Longitudinal tumor size, defined as the sum of the longest diameters of target lesions according to the RECIST criterion (23), was analyzed. Patients with at least two tumor size measurements were defined as evaluable. Two TGI models (Supplementary Material) previously proposed were fit to data from evaluable patients using NONMEM: a biexponential model (24) and a simplified TGI (sTGI) model (25). Shrinkage in parameter estimates was estimated (26). The fits of models were compared using the log-likelihood ratio test as well as standard goodness of fit plots. These models were not subjected to any simulation-based assessment since they were not meant to be used for simulation but only to estimate individual TGI metrics to be tested in the survival model. Three individual TGI metrics were calculated using individual post hoc TGI parameter estimates: the growth rate constant (KG) (24), the TTG (25), and tumor size ratio to baseline at week 8 (25). 
Table I. Patient Characteristics by Treatment

\begin{tabular}{|c|c|c|}
\hline & Onartuzumab arm & Placebo arm \\
\hline Total number of patients & 319 & 317 \\
\hline Study (OAM4558g:OAM4971g) ${ }^{\mathrm{a}}$ & $69: 250$ & $68: 249$ \\
\hline Age (year) & $63[24 \sim 83]$ & $63[27 \sim 84]$ \\
\hline Gender (female:male) ${ }^{\mathrm{a}}$ & $140: 179$ & $136: 181$ \\
\hline Body weight (kg) & $69.1[41.9 \sim 119.8]$ & $70.1[32.8 \sim 141]$ \\
\hline Asian patients ${ }^{\mathrm{b}}$ & $11.6 \%$ & $12.0 \%$ \\
\hline ECOG score $(0: 1: 2)^{\mathrm{a}}$ & $115: 196: 8$ & $99: 214: 3$ \\
\hline Stage (IIIB:IV) ${ }^{\mathrm{a}}$ & $82: 237$ & $78: 239$ \\
\hline Non-squamous:squamous ${ }^{\mathrm{a}}$ & 283:36 & $281: 36$ \\
\hline Albumin $(g / L)$ & $39[25 \sim 49]$ & 39 [24 49] \\
\hline Total protein $(\mathrm{g} / \mathrm{L})$ & $70[0.072 \sim 96]$ & 69 [6.9 94] \\
\hline Baseline tumor burden (mm) & $47.5[1 \sim 245]$ & $48[1 \sim 324]$ \\
\hline Number of metastatic sites & $3[1 \sim 8]$ & $2.5[1 \sim 9]$ \\
\hline More than one metastatic site ${ }^{\mathrm{b}}$ & $75.5 \%$ & $81 \%$ \\
\hline Liver metastasis present ${ }^{\mathrm{b}}$ & $20.7 \%$ & $20.2 \%$ \\
\hline Smoking history (current:previous:never) ${ }^{\mathrm{a}}$ & 50:209:59 & $57: 208: 50$ \\
\hline EGFR mutation (yes:no:missing) ${ }^{\mathrm{a}}$ & $43: 232: 44$ & $41: 237: 39$ \\
\hline KRAS mutation (yes:no:missing) ${ }^{\mathrm{a}}$ & $36: 80: 203$ & 27:101:199 \\
\hline MET mutation (yes:no:missing) ${ }^{\mathrm{a}}$ & 270:31:18 & $271: 31: 15$ \\
\hline Time since diagnosis (months) & $11.8[0.46 \sim 90.7]$ & $12.4[1.87 \sim 97.3]$ \\
\hline Estimate of TTG (weeks) & $1.445[-44.62 \sim 68.50]$ & $1.357[-36.68 \sim 70.49]$ \\
\hline Tumor size ratio to baseline at week 8 & $1.010[0.199 \sim 3.458]$ & $1.010[0.144 \sim 1.879]$ \\
\hline Estimate of KG (per week) & $0.0083[0.0043 \sim 0.1558]$ & $0.0083[0.0028 \sim 0.0803]$ \\
\hline
\end{tabular}

Patients in the onartuzumab arm receive both erlotinib and onartuzumab. Patients in the placebo arm received erlotinib alone. The numbers displayed represent the median (range) except for the number of patients is displayed (superscript letter a) and the percentage of patients is displayed (superscript letter b)

ECOG score Eastern Cooperative Oncology Group performance score, EGFR epidermal growth factor receptor, $K G$ growth rate constant, KRAS Kirsten rat sarcoma viral oncogene homolog, MET hepatocyte growth factor receptor, TTG time to tumor re-growth

\section{Exposure-Response Analysis of Efficacy Endpoints}

Time-to-event variables (PFS, OS, and TTG) were explored and compared between patient subgroups with different onartuzumab exposure using Kaplan-Meier analysis and logrank test. Multivariate models were developed using Cox regression analysis to associate time-to-event variables with onartuzumab exposure, clinically relevant covariates, and TGI metrics (when applicable). A "full" model was built by including all significant covariates from the Cox univariate analysis $(p<0.05$ as per the log-likelihood ratio test where the difference in $-2 * \log$-likelihood (score) between the alternative models follows a $\chi^{2}$ distribution). If several exposure or TGI metrics were significant from the univariate analysis, only the most significant one was included in the full model. Then, a backward stepwise elimination was carried out. At each elimination step, the relative influence of each remaining covariate on the model was re-evaluated one by one by deleting it from the reduced model using a cutoff of $p<0.01$. The model was built only based on the patient subset with complete information without imputation. The PFS model was developed without inclusion of TGI metrics as covariates in MET-positive patients because tumor response was already considered and included in the overall clinical assessment of PFS.

\section{Exposure-Response Analysis of Safety Endpoints}

The safety endpoints (adverse events) were characterized by frequency (yes/no). The proportions of frequency and
$95 \%$ confidence intervals were computed for intervals of onartuzumab exposure with an equivalent number of individuals (e.g., quartiles or tertiles). The correlation between each safety endpoint and onartuzumab exposure subgroups was tested using logistic regression and the Wald test. Multivariate logistic regression models were developed only for the safety endpoints that showed a statistically significant $(p<0.05)$ correlation with any onartuzumab exposure metrics. All candidate covariates and exposure metrics were tested in a univariate and multivariate manner with the significance level of $p<0.05$. The model was built only based on the patient subset with complete information without imputation.

\section{RESULTS}

\section{Patients and Exposure Metrics}

Data from a total of 636 patients were collected. Baseline patient characteristics evaluated as covariates are summarized in Table I. Both MET-positive and MET-negative patients were enrolled in OAM4558g, while only MET-positive patients were enrolled in OAM4971g. In OAM4558g, 27 patients (13 MET-positive, 12 MET-negative, and 2 unknown MET status) in the placebo arm received onartuzumab at some point (crossover patients) and thus were excluded from the analysis. Exposure data were unavailable in three patients who received onartuzumab, leaving 606 patients included in the analysis (550 MET-positive). PK parameter estimates 
based on the PK data from OAM4558g and OAM4971g were consistent with those previously reported (16).

\section{Longitudinal Tumor Size Modeling}

Two TGI models were fit to the longitudinal tumor size data. A total of 584 patients were evaluable for TGI modeling. Parameter estimates and goodness-of-fit plots for these two TGI models were summarized in Supplementary Tables 1 and 2 and Supplementary Figures 1 and 2. Both models provided adequate fit to the data with a slightly better fit by the bi-exponential model. The KG, TTG, and tumor size ratio to baseline at week 8 were derived.

\section{Exposure-Response Analysis for Survival}

The highest quartile of onartuzumab steady-state Cmin (Cmin,ss) was associated with a longer PFS (median 4.37 months) than the other three quartiles (median 2.50 months) and the placebo arm (median 2.50 months) (Fig. 2) (Supplementary Tables 3 and 4). Univariate Cox regression analysis for OS (Table II) showed a strong association between OS and TTG $(p<0.0001)$. The association between OS and tumor size ratio or log (KG) was statistically significant $(p<0.0001)$, but not as strong as TTG. The final OS model (Table III) was obtained by multivariate backward stepwise elimination. In this model, none of the exposure metrics was significant $(p>0.05)$ whether they were considered a continuous variable or a categorical variable with the upper quartile as threshold.

\section{Correlation Between Time to Tumor Re-growth and Onartuzumab Exposure}

As illustrated in Fig. 1, one possible reason why onartuzumab exposure was eliminated from the final OS model could be due to the potential association between

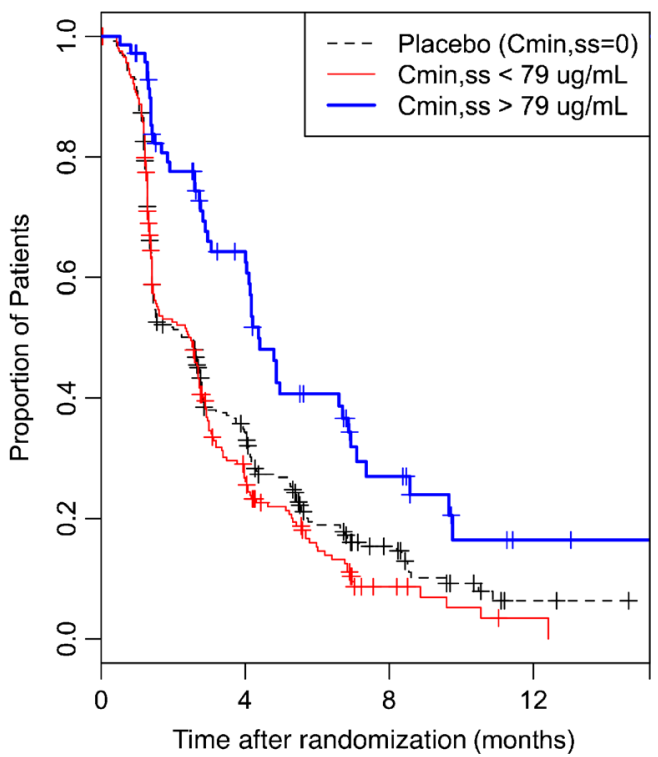

Fig. 2. Kaplan-Meier plot comparing progression-free survival by onartuzumab exposure. Cmin,ss onartuzumab steady-state trough serum concentration with an upper quartile threshold of $79 \mu \mathrm{g} / \mathrm{mL}$
Table II. Univariate Cox Regression Analysis for Overall Survival

\begin{tabular}{lllll}
\hline Covariate & Score & $p$ value & Number & Sign \\
\hline Albumin $(\mathrm{g} / \mathrm{L})$ & 26.8 & $<0.0001$ & 506 & - \\
Number of metastatic sites & 21.4 & $<0.0001$ & 587 & + \\
Estimate of TTG (weeks) & 20.5 & $<0.0001$ & 582 & - \\
Baseline tumor burden $(\mathrm{mm})$ & 16.4 & $<0.0001$ & 582 & + \\
More than one metastatic site & 14.9 & $<0.0001$ & 587 & + \\
ECOG score $>0$ & 14 & $<0.0001$ & 606 & + \\
TR to baseline at week 8 & 12.4 & $<0.0001$ & 582 & + \\
EGFR mutation positive & 11.4 & $<0.0001$ & 606 & - \\
log (KG) & 9.9 & $<0.0001$ & 582 & + \\
Gender = male & 7.5 & 0.0001 & 606 & + \\
Current smoker & 7.2 & 0.0001 & 606 & + \\
Cmin,ss $>77 \mu \mathrm{g} / \mathrm{mL}$ & 6.2 & 0.0005 & 606 & - \\
Liver metastasis present & 4.9 & 0.0017 & 606 & + \\
Time since diagnosis (month) & 4.6 & 0.0024 & 584 & - \\
Asian ethnicity & 3.2 & 0.012 & 606 & - \\
Age (year) & 2.2 & 0.0352 & 606 & - \\
Onartuzumab treatment & 0.5 & 0.3093 & 606 & + \\
OAM4558g study & 0.1 & 0.6789 & 606 & - \\
MET mutation positive & 0.1 & 0.7426 & 600 & - \\
\hline
\end{tabular}

Covariates are ordered by the level of significance (score). Covariates tested included all baseline covariates, onartuzumab exposure, and TGI (tumor growth inhibition) metrics. $p$ value is calculated using the likelihood ratio test. Score means the difference in $-2 * \log$-likelihood between the alternative models following a $\chi^{2}$ distribution. Sign is the death hazard increases $(+)$ or decreases $(-)$ with the increased value of the covariate with a positive sign indicating bad prognosis Abbreviations: Cmin,ss steady-state trough onartuzumab serum concentration with an upper quartile threshold of $77 \mu \mathrm{g} / \mathrm{mL}, E C O G$ score Eastern Cooperative Oncology Group performance score, $E G F R$ epidermal growth factor receptor, $K G$ growth rate constant, $N$ number of patients with available information of the specific covariate, $T R$ tumor size ratio to baseline, $T T G$ time to tumor regrowth

longer TTG and higher onartuzumab exposure. Therefore, this was subsequently examined by univariate and multivariate Cox regression analysis linking TTG with baseline prognostic factors and onartuzumab exposure.

The univariate Cox regression analysis for TTG is summarized in Supplementary Table 5. EGFR mutation status and baseline albumin were selected to remain in the final TTG model as independent predictors for TTG (Table III). In the final TTG model, the presence of EGFR mutation and higher albumin was associated with longer TTG. None of the onartuzumab exposure metrics was significant in the final TTG model. There was no interaction between onartuzumab Cmin,ss and MET diagnostic status.

\section{Exposure-Response of Safety Endpoints}

Seven adverse events were evaluated: infusion reactions, allergic reactions, venous thromboembolism, arterial thromboembolism, interstitial lung disease, gastrointestinal perforations, and peripheral edema. Peripheral edema data were unavailable in OAM4558g. Exploratory logistic regression showed that only infusion reactions and peripheral edema were statistically significantly associated with onartuzumab exposure (Fig. 3). The final logistic regression (Table III) was obtained by multivariate logistic regression. Among all 
Table III. Parameter Estimates of Final Models for Efficacy and Safety Endpoints

\begin{tabular}{|c|c|c|c|c|}
\hline Parameter & Estimate & RSE & $z$ & $p$ value \\
\hline \multicolumn{5}{|l|}{ Final OS model ${ }^{\mathrm{a}}$} \\
\hline Baseline albumin (g/L) & -0.09545 & 15.1 & -6.61 & $<0.0001$ \\
\hline Estimate of TTG (weeks) & -0.02413 & 17.4 & -5.73 & $<0.0001$ \\
\hline Number of metastatic sites & 0.22365 & 19.5 & 5.13 & $<0.0001$ \\
\hline Gender $=$ male & 0.5510 & 25.1 & 3.98 & $<0.0001$ \\
\hline ECOG score $>0$ & 0.4450 & 33.9 & 2.95 & 0.0032 \\
\hline Age (year) & -0.01891 & 35.2 & -2.84 & 0.0045 \\
\hline \multicolumn{5}{|l|}{ Final TTG model ${ }^{\mathrm{b}}$} \\
\hline EGFR mutation positive & -0.802 & 16.9 & -5.91 & $<0.0001$ \\
\hline Baseline albumin (g/L) & -0.0287 & 35.9 & -2.78 & 0.0054 \\
\hline \multicolumn{5}{|l|}{ Infusion reactions $\mathrm{s}^{\mathrm{c}}$} \\
\hline (Intercept) & -1.78 & 7.30 & -13.7 & $<0.0001$ \\
\hline Cmin,ss $>79 \mu \mathrm{g} / \mathrm{mL}$ & 0.915 & 31.8 & 3.14 & 0.0017 \\
\hline \multicolumn{5}{|l|}{ Peripheral Edema ${ }^{c}$} \\
\hline (Intercept) & -2.17 & 8.39 & -11.9 & $<0.0001$ \\
\hline Cmin,ss $(\mu \mathrm{g} / \mathrm{mL})$ & 0.0166 & 18.6 & 5.37 & $<0.0001$ \\
\hline
\end{tabular}

$\mathrm{p}$ value: Wald test $\left(\chi^{2}\right) . z$ : Wald statistic

Abbreviations: Cmin,ss steady-state trough onartuzumab serum concentration with an upper quartile threshold of $79 \mu \mathrm{g} / \mathrm{ml}, E C O G$ score Eastern Cooperative Oncology Group performance score, $E G F R$ epidermal growth factor receptor, $O S$ overall survival. $R S E$ relative standard error $(\%)$ indicating the precision of parameter estimation, TTG time to tumor re-growth

${ }^{a}$ Developed using multivariate Cox regression models to associate OS with all baseline covariates, onartuzumab exposure, and tumor growth inhibition metrics

${ }^{b}$ Developed using multivariate Cox regression models to associate TTG with all baseline covariates and onartuzumab exposure

${ }^{c}$ Developed using multivariate logistic regression models to associate the incidence of adverse events with all baseline covariates and onartuzumab exposure

baseline prognostic factors and onartuzumab exposure metrics tested, only onartuzumab Cmin,ss in the highest quartile was associated with increased incidence of infusion reactions, and only increased onartuzumab Cmin,ss was associated with increased incidence of peripheral edema.

\section{DISCUSSION}

The phase III trial OAM4971g comparing onartuzumab plus erlotinib vs. erlotinib in MET-positive patients with the second- and third-line NSCLC did not meet the primary endpoint of OS. This analysis was performed to assess whether higher onartuzumab doses might yield better efficacy without compromising the safety profile. Our analysis demonstrated that higher onartuzumab PK exposure was not associated with improved OS after accounting for baseline prognostic factors and TGI metrics, but there was a trend of unknown clinical significance toward increased incidence of infusion reactions and peripheral edema. These results did not support testing of a higher onartuzumab dose in this population.

Exploratory analysis suggested that patients with the onartuzumab steady-state trough concentration (Cmin,ss) above the highest quartile tended to have a longer OS than patients with Cmin,ss in the lowest three quartiles, who had
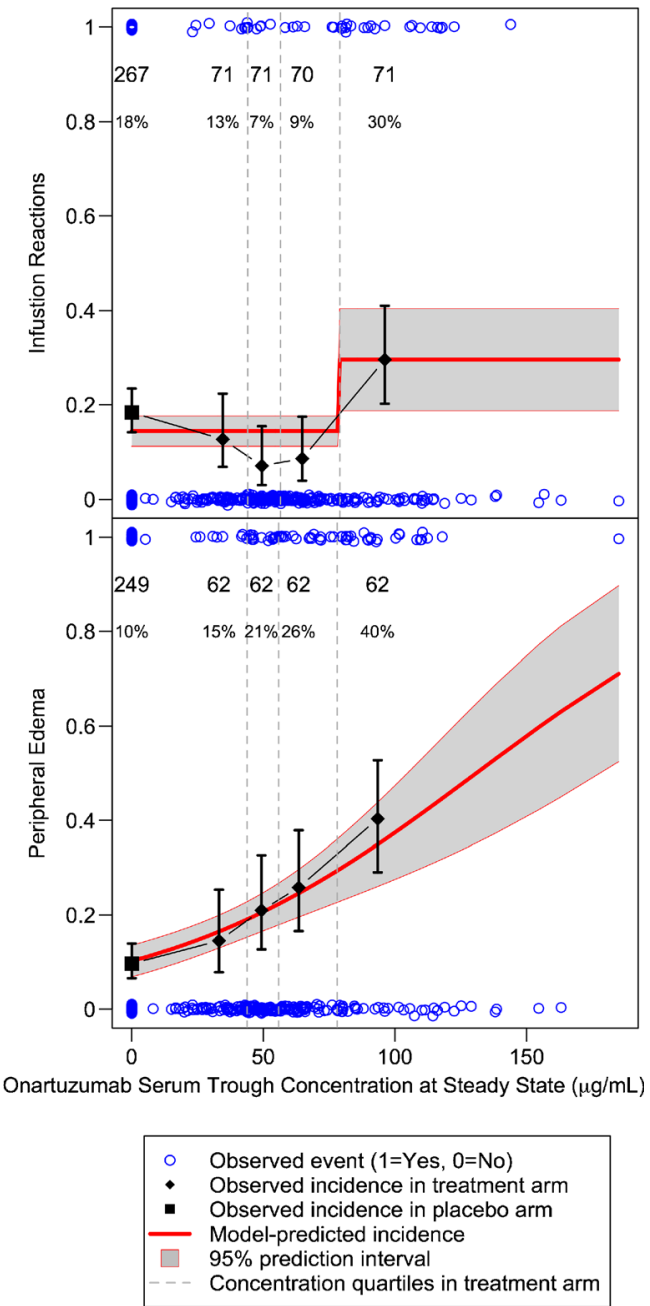

Fig. 3. Exposure-safety relationship for infusion reactions and peripheral edema. The model-predicted incidence with $95 \%$ prediction interval was generated by the final logistic regression model. Onartuzumab concentration was assumed to be zero in the placebo arm. Observed incidence was calculated with error bar in the placebo arm and in each concentration quartile in the treatment arm. The number displayed above each observed incidence was the corresponding number of patients and calculated incidence

similar or even slightly lower OS as compared to patients receiving erlotinib alone. In the final multivariate model after adjusting for baseline prognostic factors and TGI metrics, higher Cmin,ss was no longer significantly associated with longer OS.

Exposure-response models may be confounded by observed and unobserved prognostic and disease risk factors $(18,19)$. Prognostic factors may impact both OS and PK exposure (Fig. 1), especially for antibody drugs. Patients with the characteristics related to poor prognosis (e.g., low albumin and high ECOG score) have demonstrated faster clearance and thus lower exposure for large molecule drugs (27). This may be adjusted for by using multivariate models incorporating baseline prognostic factors, such as the Cox regression employed in this analysis. The resulting multivariate models may be used to simulate the expected response under unstudied doses (28). However, such simulations may lead to biased inferences if the confounding is not fully 
adjusted for. One way to address the confounding issue is to incorporate an explanatory variable, such as TGI metrics, as illustrated in the causal pathway (18) in Fig. 1. A good example has been established using data for trastuzumab in HER2-positive gastric cancer (29). The multivariate OS model accounts for both baseline prognostic factors and treatment effect on TGI. Assuming that the drug effect is mediated via TGI, the direct exposure-OS link should be removed when TGI enters the OS model, which has been the case in this analysis. If the exposure-OS link is valid, valid inferences can be made assuming exposure impacts TGI and TGI impacts OS. However, there was no exposure-TGI link in this analysis, demonstrating that a valid exposure-OS link does not exist.

In this analysis, TGI metrics were estimated based on longitudinal tumor size data (18). The estimate of time to tumor re-growth (TTG) was a strong predictor for OS as observed in previous studies using similar methodologies (18). TTG remained a strong predictor of OS after adjustment for baseline prognostic factors. In the univariate analysis for TTG, patients with the onartuzumab Cmin,ss above the highest quartile tended to have longer TTG. However, the main covariate effects were EGFR mutation status and baseline albumin with EGFR-mutated patients with higher albumin having a trend to have longer TTG. It is well known that EGFR-mutated patients respond better to erlotinib treatment than other patients, and patients with higher albumin are healthier and usually show better clinical outcomes than patients with lower albumin. Onartuzumab exposure was no longer significant after adjusting for EGFR mutation status and albumin, suggesting that onartuzumab treatment had no discernable effect on TGI and that the exposure-OS relationship observed in the exploratory analysis was mainly due to disease prognostic.

The exposure-response analysis was performed for all seven selected adverse events without distinguishing onartuzumab-related or erlotinib-related adverse events. Statistically significant relations between onartuzumab Cmin,ss and infusion reactions and peripheral edema were observed. In the multivariate analyses, no baseline prognostic factors entered the model and onartuzumab exposure was the only predictor. For infusion reactions, the incidence appeared to be increased in patients with the onartuzumab Cmin,ss above the highest quartile. In addition, the incidence was notably higher in OAM4558g than in OAM4971g, likely due to the higher onartuzumab concentrations in OAM4558g. Onartuzumab Cmin,ss in $34 \%$ of patients in OAM4558g but only $24 \%$ in OAM4971g were above the highest quartile threshold of $79 \mu \mathrm{g} / \mathrm{mL}$. However, this result should be interpreted with caution because an infusion reaction might be caused by infusion that lasted longer than was prespecified in the protocol, especially when a higher dose was given. Unfortunately, this could not be further evaluated due to the lack of information on the exact time when the infusion reaction occurred. In contrast, the incidence of peripheral edema appeared to be associated with onartuzumab exposure that was evaluated as either a continuous or categorical variable, and all onartuzumab exposure metrics were statistically significant. The clinical significance of the exposureresponse relationships for infusion reactions and peripheral edema was unclear.
There are several limitations in this analysis. First, only one dose level $(15 \mathrm{mg} / \mathrm{kg})$ was evaluated in the phase II and phase III studies, which may limit the range of exposure. However, onartuzumab Cmin,ss ranged from 5.2 to $185 \mu \mathrm{g} /$ $\mathrm{mL}$ in the pooled data, with a coefficient of variation $(\mathrm{CV})$ of $46 \%$. This may provide an adequately wide range of exposure to be tested in the model. Second, there was some shrinkage in parameter and individual estimates of the TGI metrics (Supplementary Tables 1 and 2). Shrinkage might decrease the predictive power of these metrics. However, all the metrics were still strong predictors of OS indicating that the shrinkage was not a major problem.

In conclusion, in patients with second- and third-line NSCLC, higher onartuzumab PK exposure was not associated with improved OS after accounting for baseline prognostic factors and TGI metrics, but there was a trend of unknown clinical significance toward increased incidence of infusion reactions and peripheral edema. Collectively, these results did not suggest that dose intensification would improve clinical outcomes and thus did not support further clinical investigation of a higher onartuzumab dose in this population.

\section{ACKNOWLEDGMENTS}

We acknowledge Bert Lum from Genentech Inc. for his expert review and comments.

\section{COMPLIANCE WITH ETHICAL STANDARDS}

Conflict of Interest Kelong Han, Jin Jin, Helen Winter, and Mark Stroh receive salary from Genentech and hold stocks in Roche Pharmaceuticals. In addition, Jin Jin also holds stock in Eli Lilly. Fredrik Jonsson, Pascal Chanu, and René Bruno received salary from Pharsight Consulting Services at the time of work.

Authors' Contributions All authors have contributed substantially to the conception and design of the analysis and drafting or revising the paper as well as giving final approval for submission.

Open Access This article is distributed under the terms of the Creative Commons Attribution 4.0 International License (http://creativecommons.org/licenses/by/4.0/), which permits unrestricted use, distribution, and reproduction in any medium, provided you give appropriate credit to the original author(s) and the source, provide a link to the Creative Commons license, and indicate if changes were made.

\section{REFERENCES}

1. Ma PC, Maulik G, Christensen J, et al. c-Met: structure, functions and potential for therapeutic inhibition. Cancer Metastasis Rev. 2003;22:309-25.

2. Schmidt L, Junker K, Nakaigawa N, et al. Novel mutations of the MET proto-oncogene in papillary renal carcinomas. Oncogene. 1999;18:2343-50.

3. Olivero M, Valente G, Bardelli A, et al. Novel mutation in the ATP-binding site of the MET oncogene tyrosine kinase in a HPRCC family. Int J Cancer. 1999;82:640-3. 
4. Ichimura E, Maeshima A, Nakajima T, et al. Expression of cmet/HGF receptor in human non-small cell lung carcinomas in vitro and in vivo and its prognostic significance. Jpn J Cancer Res. 1996;87:1063-9.

5. Koochekpour S, Jeffers M, Rulong S, et al. Met and hepatocyte growth factor/scatter factor expression in human gliomas. Cancer Res. 1997;57:5391-8.

6. Wu CW, Li AF, Chi CW, et al. Hepatocyte growth factor and Met/HGF receptors in patients with gastric adenocarcinoma. Oncol Rep. 1998;5:817-22.

7. Turesson I, Abildgaard N, Ahlgren T, et al. Prognostic evaluation in multiple myeloma: an analysis of the impact of new prognostic factors. Br J Haematol. 1999;106:1005-12.

8. Uchida D, Kawamata H, Omotehara F, et al. Role of HGF/cMet system in invasion and metastasis of oral squamous cell carcinoma cells in vitro and its clinical significance. Int J Cancer. 2001;93:489-96.

9. Baykal C, Ayhan A, Al A, et al. Overexpression of the c-Met/ HGF receptor and its prognostic significance in uterine cervix carcinomas. Gynecol Oncol. 2003;88:123-9.

10. Masuya D, Huang C, Liu D, et al. The tumour-stromal interaction between intratumoral c-Met and stromal hepatocyte growth factor associated with tumour growth and prognosis in non-small-cell lung cancer patients. Br J Cancer. 2004;90:1555-62.

11. Ayhan A, Ertunc D, Tok EC, et al. Expression of the c-Met in advanced epithelial ovarian cancer and its prognostic significance. Int J Gynecol Cancer. 2005;15:618-23.

12. Cheng HL, Liu HS, Lin YJ, et al. Co-expression of RON and MET is a prognostic indicator for patients with transitional-cell carcinoma of the bladder. Br J Cancer. 2005;92:1906-14.

13. Lengyel E, Prechtel D, Resau JH, et al. c-Met overexpression in nodepositive breast cancer identifies patients with poor clinical outcome independent of Her2/neu. Int J Cancer. 2005;113:678-82.

14. Spigel D, Ervin T, Ramlau R, et al. Randomized phase II trial of onartuzumab in combination with erlotinib in patients with advanced non-small-cell lung cancer. J Clin Oncol. 2013;31:4105-14.

15. Spigel DR, Edelman MJ, et al. Onartuzumab plus erlotinib versus erlotinib in previously treated stage IIIb or IV NSCLC: results from the pivotal phase III randomized, multicenter, placebo-controlled METLung (OAM4971g) global trial. J Clin Oncol. 2014;32(suppl; abstr 8000):5s.

16. Xin Y, Jin D, Eppler S, et al. Population pharmacokinetic analysis from phase I and phase II studies of the humanized monovalent antibody, onartuzumab (MetMAb), in patients with advanced solid tumors. J Clin Pharmacol. 2013;52:1103-11.
17. Salgia R, Patel P, Bothos J, et al. Phase I dose-escalation study of onartuzumab as a single agent and in combination with bevacizumab in patients with advanced solid malignancies. Clin Cancer Res. 2014;20(6):1666-75.

18. Bruno R, Mercier F, Claret L. Evaluation of tumor-size response metrics to predict survival in oncology clinical trials. Clin Pharmacol Ther. 2014;95:386-93.

19. Yang $\mathrm{J}$ et al. The combination of exposure-response and casecontrol analyses in regulatory decision making. J Clin Pharmacol. 2013;53:160-6.

20. Beal S, Sheiner LB, Boeckmann A, Bauer RJ. NONMEM user's guides. (1989-2009), Icon Development Solutions, Ellicott City, MD, USA; 2009.

21. Lindbom L, Ribbing J, Jonsson EN. Perl-speaks-NONMEM (PsN) - a Perl module for NONMEM related programming. Comput Methods Prog Biomed. 2004;75(2):85-94.

22. R Core Team. R: A language and environment for statistical computing. R Foundation for Statistical Computing, Vienna, Austria. 2014. URL: http://www.R-project.org/.

23. Eisenhauer EA, Therasse P, Bogaerts J, et al. New response evaluation criteria in solid tumors: revised RECIST guideline (version 1.1). Eur J Cancer. 2009;45:228-47.

24. Stein WD, Gulley JL, Schlom J, et al. Tumor regression and growth rates determined in five intramural NCI prostate cancer trials: the growth rate constant as an indicator of therapeutic efficacy. Clin Cancer Res. 2010;17:907-17.

25. Claret L, Gupta M, Han K, et al. Evaluation of tumor size response metrics to predict overall survival in western and Chinese patients with first line metastatic colorectal cancer. J Clin Oncol. 2013;31:2110-4.

26. Savic RM, Karlsson MO. Importance of shrinkage in empirical Bayes estimates for diagnostics: problems and solutions. AAPS J. 2009;11:558-69.

27. Dostalek M, Gardner I, Gurbaxani BM, Rose RH, Chetty M. Pharmacokinetics, pharmacodynamics and physiologicallybased pharmacokinetic modelling of monoclonal antibodies. Clin Pharmacokinet. 2013;52:83-124.

28. Lu JF et al. Exposure-response relationship of AMG 386 in combination with weekly paclitaxel in recurrent ovarian cancer and its implication for dose selection. Cancer Chemother Pharmacol. 2012;69:1135-44.

29. Quartino AL, et al. Evaluation of tumor size metrics to predict survival in advanced gastric cancer (abstr 2812). 2013. http://www.page-meeting.org/?abstract=2812. 